\title{
基于偶氮苯衍生物的三阶非线性光开关性能的调控
}

\author{
翟亚丽许文娟孟祥茹* 侯红卫* \\ (郑州大学化学学院 郑州 450001)
}

\begin{abstract}
摘要 偶氮苯衍生物因其具有特殊的共轭体系常用作光开关特性的三阶非线性光学(NLO)开关材料, 但有部分带有特 殊基团的偶氮类分子在一般条件下无法显示其性能. 然而，这类分子在被调节之后能展示出优异的光控的 NLO 开关性 能. 以无光控的 NLO 开关性能的 5-(N-4-偶氮苯基)氨甲基间苯二甲酸为研究对象, 并对其潜在的光控 NLO 开关性能进 行调控. 经过调节后的这类分子在光照条件下能顺利地经历可逆的顺反异构化反应，且产生三阶非线性性能的转换. 其 Z-扫描实验结果显示处于反式构型的材料展示出反饱和吸收和自散焦特性; 在光照之后, 这类材料转化为顺式构型 并展现出饱和吸收和自聚焦行为. 三阶 NLO 性能的转换是由于材料的结构发生转变其内部电子产生重排, 使得它们在 激光刺激下产生不同的响应机制.
\end{abstract}

关键词 顺反异构; 光控的; 三阶 NLO 开关; 质子效应; 性能转变

\section{Adjusting the Third-Order Nonlinear Optical Switch Performance Based on Azobenzene Derivatives}

\author{
Zhai, Yali $\quad \mathrm{Xu}$, Wenjuan $\quad$ Meng, Xiangru* Hou, Hongwei* \\ (College of Chemistry, Zhengzhou University, Zhengzhou 450001)
}

\begin{abstract}
The photo-controllable third-order nonlinear optical (NLO) switches have drawn ever-increasing attention due to considerable research potential in the emerging field of nonlinear optics. A class of materials, which contain photosensitive groups but cannot express directly switching properties under light conditions, can also exhibit the characteristics of excellent photo-controllable NLO switches after external regulation. Azobenzene is a kind of classic photo-isomerized molecule and has good $\pi$ coplanar property and excellent electron channel, which can engender third-order NLO response under push and pull electron action. It is a feasible strategy to design photo-controlled NLO switch materials by introducing azo groups. Nevertheless, the trans-cis isomerization behaviors of some azobenzene derivatives are interfered by other groups or external factors, further inhibiting the conversion of photo-controllable third-order NLO properties. Once these external interference factors are found and removed, the photo-controllable NLO behaviors of such azobenzene derivatives will be opened. In our work, a special azobenzene derivative was synthesized and reported, which was unable to produce cis-trans isomerization due to the $\mathrm{H}^{+}$effect of self-dissociation, and the $\mathrm{H}^{+}$effect could be shield by introducing organic groups or bases. The adjusted materials can easily undergo reversible cis-trans isomerization reaction, and the Z-scan test shows the complete inversions of third-order NLO properties before and after UV irradiation. The adjusted materials in trans configuration show the reverse saturation absorption (RSA) and self-defocusing properties. After UV irradiation, the materials convert into cis configuration and exhibit saturation absorption (SA) and strong self-focusing behaviors. To gain a deeper understanding of the light-adjusted third-order NLO switch behaviors, density functional theory (DFT) calculations of $\left(\mathrm{CH}_{3}\right)_{2} \mathrm{~L}$ were carried out. For trans- $\left(\mathrm{CH}_{3}\right)_{2} \mathrm{~L}, \mathrm{HOMO}$ and LUMO are mainly localized on the azobenzene unit, where $\pi-\pi^{*}$ transition between the two orbitals is displayed. The azobenzene unit in the trans- $\left(\mathrm{CH}_{3}\right)_{2} \mathrm{~L}$ is considered to have considerable contribution to the generation of third-order nonlinearity. For cis- $\left(\mathrm{CH}_{3}\right)_{2} \mathrm{~L}$, the electron cloud density of HOMO is mainly populated on the azobenzene unit, whereas the electron cloud density distribution of LUMO appears on the entire molecule, suggesting significant intramolecular charge transfer (ICT) from azobenzene to the entire molecule. The effect of ICT in the cis structure dominants the generation of third-order nonlinearity. The remarkable third-order NLO transformation result from the rearrangement of the electronic structures, which makes them generate different response mechanisms under the laser stimulation.
\end{abstract}

Keywords cis-trans isomerization; photo-controllable; third-order NLO switch; protons effect; performance transformation

\section{1 引言}

传统的光控三阶非线性光学开关材料易于在两种
不同的化学形式之间进行转变, 进而在外界光刺激下产 生可逆性三阶非线性光学(NLO)性能的变化 ${ }^{[1 \sim 3]}$. 这类

\footnotetext{
*E-mail: mxr@zzu.edu.cn; houhongw@zzu.edu.cn
}

Received December 16, 2019; published March 5, 2020.

Supporting information for this article is available free of charge via the Internet at http://sioc-journal.cn.

Project supported by the National Natural Science Foundation of China (No. 21671174), the Zhongyuan Thousand Talents Project and the Natural Science Foundation of Henan Province (No. 182300410008).

项目受国家自然科学基金(21671174)、中原千人计划和河南省自然科学基金(182300410008)资助. 
材料因其在新兴的非线性光学领域具有巨大的研究潜 力而受到越来越多的关注 ${ }^{[4 \sim 7]}$. 这类材料被称为光控的 三阶非线性光学开关. 与之相反, 还存在另一类含有光 敏性基团却不能直接表达其光开关特性的材料 ${ }^{[8,9]}$, 这 就需要通过外界进行调节才能展示出光控非线性光学 开关特性. 通常, 这类需要调节才能展示出光开关特性 的材料不被认为是光控的非线性光学开关, 因此一直被 忽略. 事实上, 这类材料被调节后, 也能展示出优良的 NLO 开关性能. 所以对这类材料的调节将会为 NLO 开 关领域开辟出一个新的方向.

偶氮苯类化合物是一类经典的光异构化分子 ${ }^{[10 ~ 13], ~}$ 且具有良好的 $\pi$ 共轭性和优良的电子通道, 能在推拉电 子作用下产生三阶 NLO 响应 ${ }^{[14 ~ 17]}$. 前人的研究表明通 过引入偶氮苯基团来设计光控 NLO 开关材料是一种可

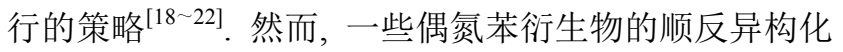
行为常常受到其自身结构特性的干扰 ${ }^{[23 ~ 25]}$, 进一步阻 碍了光控三阶 NLO 性质的转换. 找到这些外部干扰因 素并把它屏蔽掉, 那么这类偶氮苯衍生物屏蔽着的光控 NLO 开关行为就会被打开(图 1). 报道了一种需要被调 节才能展示光控 NLO 开关材料 5-( N-4-偶氮苯基)氨甲基 间苯二甲酸 $\left(\mathrm{H}_{2} \mathrm{~L}\right)$, 通过引入有机基团或碱去屏蔽 $\mathrm{H}^{+}$的 影响对其材料进行调控. 令人满意的是, 当调节后的材 料被 UV 照射后, 三阶 NLO 性能的响应产生了从反饱和 吸收到饱和吸收以及从自散焦到强的自聚焦的转变. 据 我们所知，这是第一例从去屏蔽的角度考虑对被 “隐藏” 的光控三阶 NLO 开关材料进行调控, 调节后的材料在 紫外光照射下其三阶 NLO 性能产生了转变. 这项工作 为开发 NLO 开关材料提供了更灵活且可行的选择.

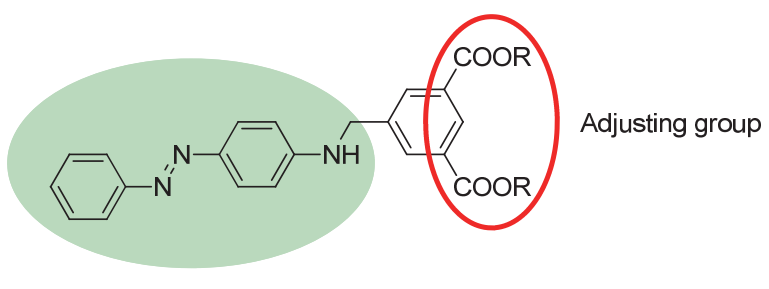

Photo-responsive group

图 1 调节 $\mathrm{R}_{2} \mathrm{~L}$ 的开关行为

Figure 1 Adjusting switch behavior of $\mathrm{R}_{2} \mathrm{~L}$

\section{2 结果与讨论}

\section{1 顺反异构化干扰因素的研究}

根据之前的报道, 偶氮苯及其衍生物在紫外光照射 下可以进行反式构型到顺式构型的转变, 而在黑暗条件 或可见光下则会发生相反的过程 ${ }^{[10,26]}$. 反式构型通常具 有更低的能量, 因此自然界中的偶氮类化合物通常处于 较为稳定的反式构型. 此外, 从反式到顺式的转变还伴 随着紫外/可见吸收光谱的变化 ${ }^{[27]}$. 为了深入研究 $\mathrm{H}_{2} \mathrm{~L}$ 在二甲基亚砜(DMSO)溶液中的光响应行为, 进行了紫
外/可见吸收光谱实验. 如图 2a 所示, $\mathrm{H}_{2} \mathrm{~L}$ 在 $410 \mathrm{~nm}$ 处 有强的特征吸收峰, 这与反式偶氮苯单元的 $\pi-\pi$ *跃迁是 一致的. 然而, 反式异构体的 $n-\pi *$ 跃迁吸收峰很弱, 完 全掩埋在 $\pi-\pi^{*}$ 跃迁之下, 只展现出一个长尾波 ${ }^{[28]}$. 在紫 外光照射 $30 \mathrm{~min}$ 后, $410 \mathrm{~nm}$ 处的吸光度保持不变, 这意 味着没有顺式构型的产物出现. $\mathrm{H}_{2} \mathrm{~L}$ 在光照前后其三阶 NLO 性质相同, 仅仅展现出弱的反饱和吸收, 无折射信 号(图 2b). 综上结果表明, $\mathrm{H}_{2} \mathrm{~L}$ 不能被用作光控的 NLO 开关材料.
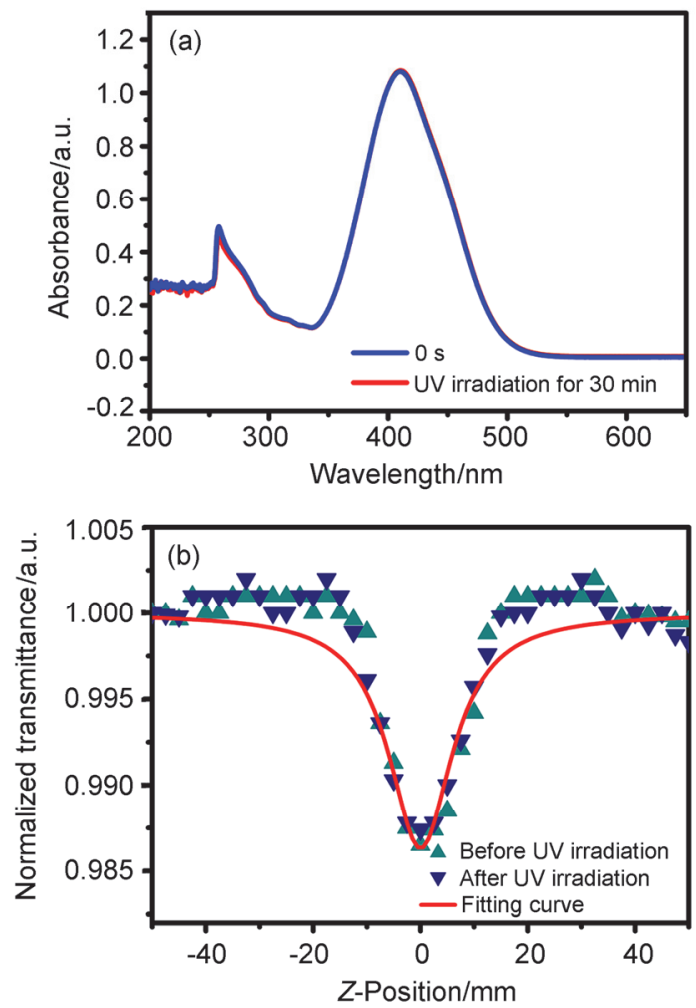

图 2 紫外光照射前后 $\mathrm{H}_{2} \mathrm{~L}$ 的紫外-可见吸收光谱(a)和 Z-扫描曲线(b) Figure 2 UV-vis absorption spectra (a) and Z-scan (b) of $\mathrm{H}_{2} \mathrm{~L}$ before and after UV irradiation

通过对分子的结构进行分析, 推测 $\mathrm{H}_{2} \mathrm{~L}$ 在溶液中解 离出的质子在顺反异构化反应中起重要作用. $\mathrm{H}_{2} \mathrm{~L}$ 溶解 在 DMSO 溶液中会部分解离 $\mathrm{H}^{+}$. 质子与反式或顺式的 偶氮基团的氮原子相互作用 ${ }^{[23 ~ 25]}$ ，质子化的氮原子将 阻止偶氮苯衍生物从反式到顺式异构化的转变，也会使 顺式构型立即返回到反式构型 ${ }^{[29]}$. 这里可能有两种途 径可以阻止 $\mathrm{H}_{2} \mathrm{~L}$ 的顺反异构化(Scheme 1).

首先 $\mathrm{H}^{+}$与部分反式 $\mathrm{H}_{2} \mathrm{~L}$ 的氮原子作用得到了中间 体 $\mathbf{A}$ ，紧接着通过分子内 $\mathrm{H}^{+}$的转移形成中间体 $\mathbf{B}$ ，中间 体 $\mathbf{A}$ 和 $\mathbf{B}$ 都对紫外光无响应; 另一方面, 不与质子结合 的反式 $\mathrm{H}_{2} \mathrm{~L}$ 能产生顺反异构化并形成顺式 $\mathrm{H}_{2} \mathrm{~L}$, 随后立 即与质子作用形成了中间体 $\mathbf{C}$, 并迅速地转化为中间体 $\mathbf{E}$, 然后中间体 $\mathbf{E}$ 通过质子转移重新恢复到最初的 $\mathrm{H}_{2} \mathrm{~L}$ 状态. 即使顺式异构体产生, 溶液中游离的 $\mathrm{H}^{+}$会立即 将顺式结构催化为反式结构. 重要的是, 其转化的速度 
非常快. 换句话说, 顺式构型的分子不能存在于 DMSO 溶液中，进而在紫外光作用下无法展现出三阶 NLO 开 关特性. 溶液中的自由质子对 $\mathrm{H}_{2} \mathrm{~L}$ 的光响应行为有着至 关重要的影响. 因此, 我们推测如果去除质子, 有可能 会实现这种材料的顺反异构化的发生.

\section{2 光控三阶非线性光开关}

为了验证去质子化的方法能实现 $\mathrm{H}_{2} \mathrm{~L}$ 的顺反异构 化, 我们合成了酯类化合物 $\left(\mathrm{CH}_{3}\right)_{2} \mathrm{~L}$, 并研究了它的光
响应行为. 单晶 $\mathrm{X}$ 射线衍射分析表明，在有机物 $\left(\mathrm{CH}_{3}\right)_{2} \mathrm{~L}$ 中，氧和 $\mathrm{CH}_{3}$ 之间存在共价键. 并且该酯的偶氮苯单元 为反式构型(图 3a). 在 $\left(\mathrm{CH}_{3}\right)_{2} \mathrm{~L}$ 的 ${ }^{1} \mathrm{H}$ NMR 谱中, $\mathrm{CH}_{3}$ 的 化学位移为 $\delta 3.89$, 进一步说明氧与 $\mathrm{CH}_{3}$ 之间存在共价 键(图 $\mathrm{S} 1$ ). 高分辨质谱显示 $\left(\mathrm{CH}_{3}\right)_{2} \mathrm{~L}$ 的分子离子峰为 404.1611 , 这与单晶 $\mathrm{X}$ 射线衍射结果相同. 同时 $\left(\mathrm{CH}_{3}\right)_{2} \mathrm{~L}$ 在 DMSO 溶液中的吸收峰出现在 $410 \mathrm{~nm}$ 处，进一步证 明了 $\left(\mathrm{CH}_{3}\right)_{2} \mathrm{~L}$ 的反式构型.

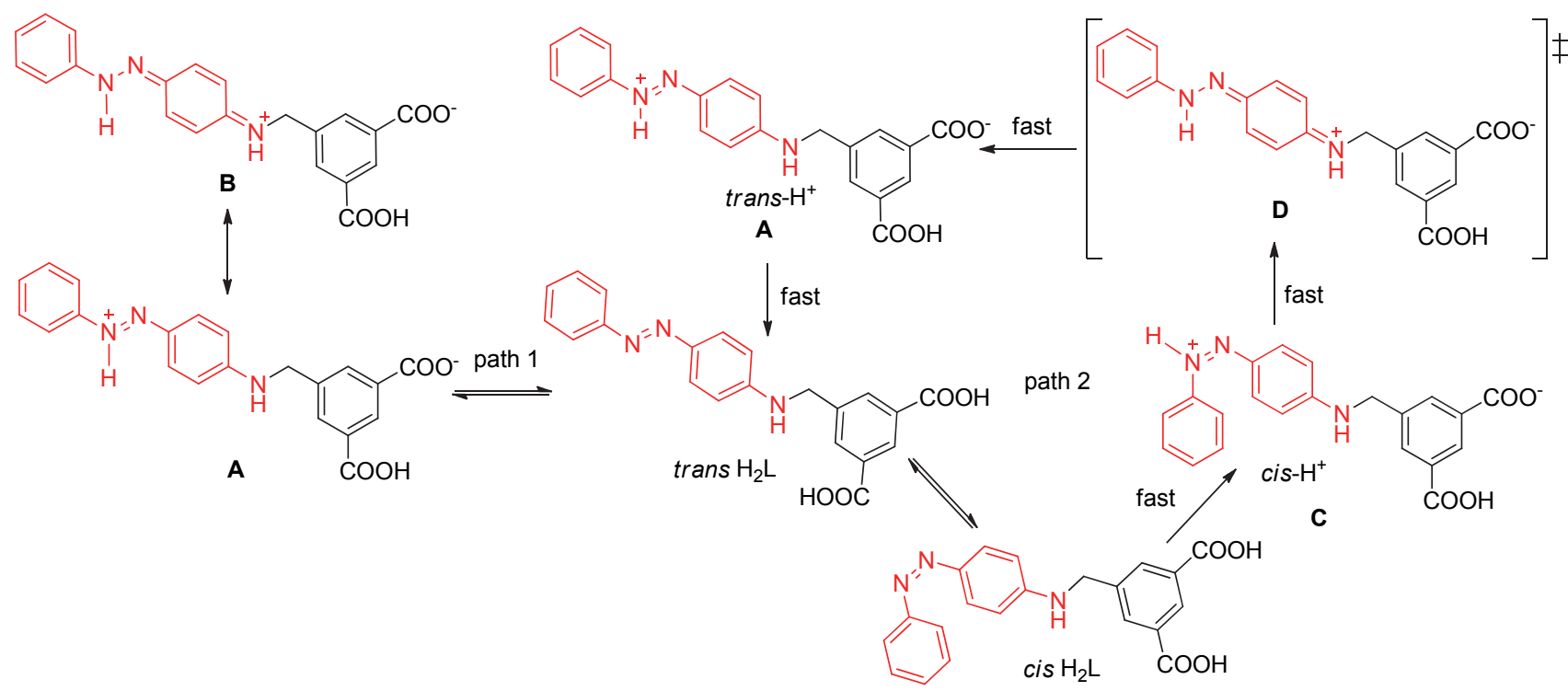

图式 1 溶解在 DMSO 溶液中的 $\mathrm{H}_{2} \mathrm{~L}$ 不能显示顺反异构化过程的机理

Scheme 1 Mechanism of the inability to show cis-trans isomerization process for $\mathrm{H}_{2} \mathrm{~L}$ dissolved in DMSO solution

(a)

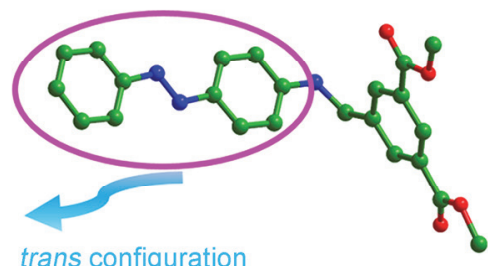

trans configuration

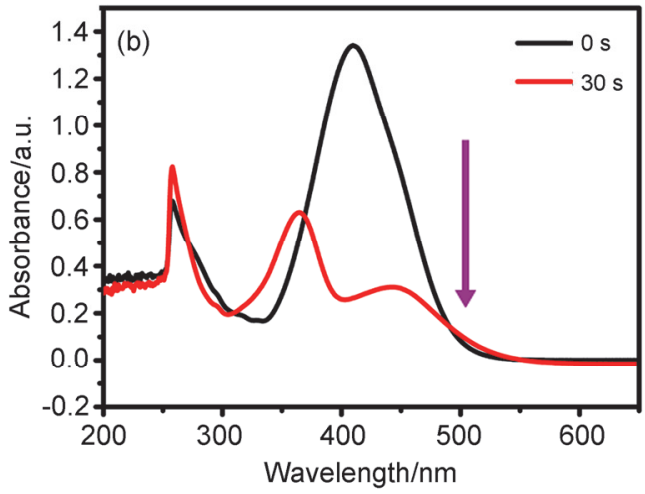

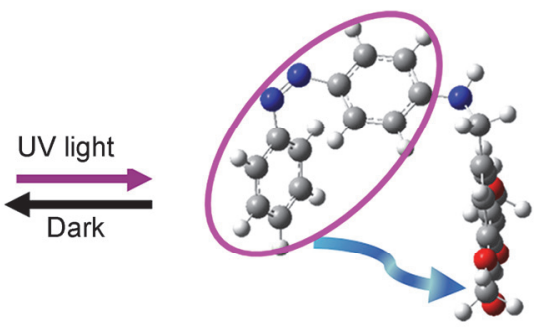

cis configuration

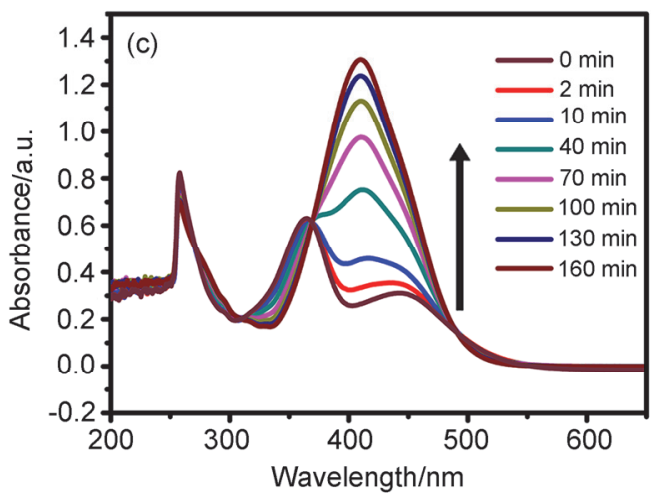

图 $3\left(\mathrm{CH}_{3}\right)_{2} \mathrm{~L}$ 的反式和顺式构型(a)、紫外光照射前后 $\left(\mathrm{CH}_{3}\right)_{2} \mathrm{~L}$ 溶液的紫外-可见吸收光谱(b)和 $\left(\mathrm{CH}_{3}\right)_{2} \mathrm{~L}$ 在黑暗中的光异构化转化过程 (c)

Figure 3 Trans and cis configurations of $\left(\mathrm{CH}_{3}\right)_{2} \mathrm{~L}$ (a), the UV-Vis absorption spectra of $\left(\mathrm{CH}_{3}\right)_{2} \mathrm{~L}$ solution before and after UV irradiation (b) and the photoisomerization conversion process of $\left(\mathrm{CH}_{3}\right)_{2} \mathrm{~L}$ in the darkness (c) 
正如所预料的那样，随着光照时间的增加 $\left(\mathrm{CH}_{3}\right)_{2} \mathrm{~L}$ 的吸收光谱发生了很大的变化. $\left(\mathrm{CH}_{3}\right)_{2} \mathrm{~L}$ 中 $410 \mathrm{~nm}$ 处的 $\pi-\pi$ *吸收强度明显下降, 并在 $365 \mathrm{~nm}$ 处出现新的吸收 峰, 这意味着 $\left(\mathrm{CH}_{3}\right)_{2} \mathrm{~L}$ 产生了反式到顺式的转变(图 3b). 原始吸收峰强度的降低以及新的吸收峰的出现是光异 构化的有力证据 ${ }^{[30,31]}$. 顺式构型的 $\left(\mathrm{CH}_{3}\right)_{2} \mathrm{~L}$ 可以在黑暗 下返回到反式构型(图 3c). 我们确认, 质子的除去使 $\mathrm{H}_{2} \mathrm{~L}$ 表现出良好的光响应行为.

鉴于上述的研究, 我们还想知道是否有可能通过引 入碱使 $\mathrm{H}_{2} \mathrm{~L}$ 成为一种光控材料. 在含有 $\mathrm{H}_{2} \mathrm{~L}$ 的 DMSO 溶液中加入 $\mathrm{K}_{2} \mathrm{CO}_{3}, \mathrm{Na}_{2} \mathrm{CO}_{3}, \mathrm{Cs}_{2} \mathrm{CO}_{3}, \mathrm{Cd}(\mathrm{Ac})_{2}, \mathrm{NH}_{3} \cdot \mathrm{H}_{2} \mathrm{O}$ 及三乙胺(TEA)等碱, 得到脱质子产物 $\mathrm{X}_{2} \mathrm{~L}$, 然后分别 对 $\mathrm{X}_{2} \mathrm{~L}$ 进行光响应实验. 以三乙胺为例, UV 照射 $30 \mathrm{~s}$ 后, 在 $420 \mathrm{~nm}$ 处对应于反式异构体的吸收峰大幅度降 低, 在 $370 \mathrm{~nm}$ 处出现了一个新的顺式异构体的吸收峰, 展现出明显的从反式到顺式的转变过程 (图 $\mathrm{S} 2 \mathrm{a}$ ). $(\mathrm{TEA})_{2} \mathrm{~L}$ 的顺式到反式的回复过程表明其具有良好的顺 反异构化能力(图 $\mathrm{S} 2 \mathrm{~b}$ ). 以上结果表明, $\mathrm{H}^{+}$在 $\mathrm{H}_{2} \mathrm{~L}$ 的顺反 异构化反应中起着极其重要的作用. 令人兴奋的是质子 的除去可以调节 $\mathrm{H}_{2} \mathrm{~L}$ 的顺反异构化. 也就是说, 分别通 过引入有机基团以及碱, 可以将 $\mathrm{H}_{2} \mathrm{~L}$ 调节成光控开关材 料, 这将为随后进一步研究光控三阶 NLO 开关行为奠 定坚实的基础.

采用顶帽 $\mathrm{Z}$ 扫描技术研究了紫外光照射前后 $\left(\mathrm{CH}_{3}\right)_{2} \mathrm{~L}$ 和 $\mathrm{X}_{2} \mathrm{~L}$ 在 DMSO 中的三阶 NLO 行为. 该仪器 利用聚焦高斯光束的样品透过率作为样品位置(Z)的因 变量, 可以直接检测出样品的 NLO 信号. 值得注意的 是, 当这些样品同时具有 NLO 吸收和折射时, 将闭孔条 件下的 Z扫描数据除以相应的开孔条件下的 Z 扫描数据 得到 NLO 折射曲线 ${ }^{[32,33]}$. 在实验中, 所有的样品浓度都 是 $1 \times 10^{-3} \mathrm{~mol} / \mathrm{L}$. 在相同的实验条件下, 首先对纯 DMSO 在光照前后的非线性性能进行测试, 排除了溶剂 产生的 NLO 的可能(图 S3). 其结果表明, DMSO 的三阶 NLO 吸收和折射基本可以忽略.

反式 $\left(\mathrm{CH}_{3}\right)_{2} \mathrm{~L}$ 在开孔条件下的 Z-扫描实验的结果见 图 4a. 归一化透过率在焦点处最小, 说明反式 $\left(\mathrm{CH}_{3}\right)_{2} \mathrm{~L}$ 具有明显的反饱和吸收行为 ${ }^{[34]}$. 反式 $\left(\mathrm{CH}_{3}\right)_{2} \mathrm{~L}$ 在闭孔条 件下的 Z-扫描曲线表明, 谷和峰是对称聚焦的, 峰到谷 表明了非线性自散焦行为 ${ }^{[35]}$, 对应于非线性负折射(图 $4 \mathrm{~b})$. 当暴露在 UV 下, 反式 $\left(\mathrm{CH}_{3}\right)_{2} \mathrm{~L}$ 转变成顺式 $\left(\mathrm{CH}_{3}\right)_{2} \mathrm{~L}$. 顺式 $\left(\mathrm{CH}_{3}\right)_{2} \mathrm{~L}$ 展现出明显的饱和吸收以及强的自聚焦行 为 $^{[36]}$. 两种构型展现出完全相反的三阶 NLO 行为. 通 过将实验数据与理论数据进行拟合, 反式 $\left(\mathrm{CH}_{3}\right)_{2} \mathrm{~L}$ 的三 阶 NLO 吸收系数 $\beta$ 是 $5.5 \times 10^{-12} \mathrm{~m} \cdot \mathrm{W}^{-1}$, 其计算非线性 折射率 $n^{2}$ 是 $-1.5 \times 10^{-18} \mathrm{~m}^{2} \cdot \mathrm{W}^{-1}$. 顺式 $\left(\mathrm{CH}_{3}\right)_{2} \mathrm{~L}$ 的 $\beta$ 值 计算得到的是 $-1.45 \times 10^{-11} \mathrm{~m} \cdot \mathrm{W}^{-1}, n^{2}$ 值是 $4.5 \times 10^{-18}$ $\mathrm{m}^{2} \cdot \mathrm{W}^{-1}$. 比较反式构型材料和顺式构型材料的 $\beta$ 和 $n^{2}$ 值, 发现样品在紫外线照射后其三阶 NLO 性质不仅发 生了转变, 而且其三阶非线性信号大大提高.
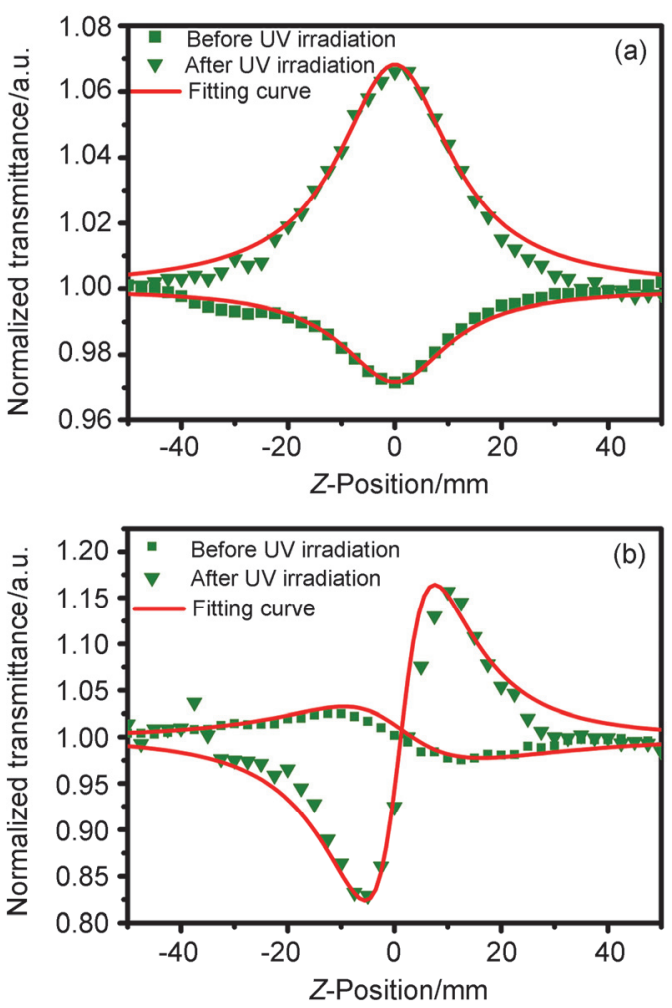

图 4 紫外光照射前和后 $\left(\mathrm{CH}_{3}\right)_{2} \mathrm{~L}$ 的三阶 $\mathrm{NLO}$ 吸收(a)和折射(b)特性 Figure 4 Third-order NLO absorption (a) and refraction (b) properties of $\left(\mathrm{CH}_{3}\right)_{2} \mathrm{~L}$ before and after UV irradiation

反式和顺式异构体在紫外-可见吸收光谱中其特征 吸收峰峰位置的不同表明其电子跃迁能级不同，进一步 影响其三阶 NLO 的性质 ${ }^{[37]}$. 为了更加深入地理解光控 的三阶 NLO 开关行为, 我们利用 Gaussian 09 软件对 $\left(\mathrm{CH}_{3}\right)_{2} \mathrm{~L}$ 进行密度泛函理论(DFT)计算, 并在 B3LYP/6$31 \mathrm{G}(\mathrm{d}, \mathrm{p})$ 水平对结构进行优化. $\left(\mathrm{CH}_{3}\right)_{2} \mathrm{~L}$ 的最高占据分子 轨道(HOMO)和最低未占据分子轨道(LUMO)的轨道分 布如图 S4 所示. 在先前的报道中认为, 当分子从 HOMO 激发到 LUMO, 存在电荷转移和 $\pi-\pi^{*}$ *跃迁 ${ }^{[38]}$, 这 两个都可以主导三阶 NLO 的产生 ${ }^{[39 \sim 41]}$. 对于反式 $\left(\mathrm{CH}_{3}\right)_{2} \mathrm{~L}, \mathrm{HOMO}$ 和 LUMO 的电子云主要坐落于偶氮苯 单元上, 两个轨道之间展现出 $\pi-\pi$ *跃迁. 反式 $\left(\mathrm{CH}_{3}\right)_{2} \mathrm{~L}$ 中的偶氮苯单元被认为对三阶非线性的产生有相当大 的贡献 ${ }^{[42]}$. 对于顺式 $\left(\mathrm{CH}_{3}\right)_{2} \mathrm{~L}, \mathrm{HOMO}$ 的电子云主要分 布在偶氮苯单元上，而 LUMO 的电子云则分布在整个 分子上, 表明从偶氮苯单元到整个分子之间存在显著的 电荷转移(ICT). 顺式结构的 ICT 效应决定了三阶非线 性的产生. 总之, 紫外光使得材料的结构发生了改变, 并导致其前沿轨道的电子云产生了重排. 这两种异构体 的三阶 NLO 性质来自于不同的机制，对应于相反的三 阶 NLO 性质.

至此, 已经知道从 $\mathrm{H}_{2} \mathrm{~L}$ 中解离出来的质子会影响顺 反异构的表达, 碱的加入会使 $\mathrm{H}_{2} \mathrm{~L}$ 具有光开关的性能. 接下来还研究了不同碱对 $\mathrm{H}_{2} \mathrm{~L}$ 的 NLO 开关性能的影响. 
分别将 $\mathrm{K}_{2} \mathrm{CO}_{3}, \mathrm{Na}_{2} \mathrm{CO}_{3}, \mathrm{Cs}_{2} \mathrm{CO}_{3}, \mathrm{Cd}(\mathrm{Ac})_{2}, \mathrm{NH}_{3} \cdot \mathrm{H}_{2} \mathrm{O}$ 和三 乙胺(TEA)等加入到 $\mathrm{H}_{2} \mathrm{~L}$ 溶液中, 并研究它们的三阶 NLO 开关特性. 因其三阶非线性光照前后转变结果一 致(图 S5), 这里以三乙胺为例进行了详细的说明. 在 $\mathrm{H}_{2} \mathrm{~L}$ 溶液中逐渐增加三乙胺的量, 在紫外光照射下捕捉 到三阶 NLO 从反饱和吸收到饱和吸收的转变过程, 并 显示出从无折射信号到产生强的自聚焦的现象. $\mathrm{H}_{2} \mathrm{~L}$ 在 没有三乙胺的情况下呈现反饱和吸收, 无折射现象. 当 该溶液中三乙胺的量达到 1.0 equiv. 时, 产生了饱和吸收 和自聚焦现象. 当三乙胺在溶液中的含量达到 2.0 equiv. 时, 饱和吸收和自聚焦信号均达到最强(图 5). 脱质子产 物 $(\mathrm{TEA})_{2} \mathrm{~L}$ 展示出优异的 NLO 性质的转变. 顺式 $(\mathrm{TEA})_{2} \mathrm{~L}$ 的三阶 $\mathrm{NLO}$ 吸收系数 $\beta$ 是 $-1.43 \times 10^{-11} \mathrm{~m} \cdot$ $\mathrm{W}^{-1}$, 其计算得到非线性折射率 $n^{2}$ 是 $3.2 \times 10^{-18} \mathrm{~m}^{2}$. $\mathrm{W}^{-1}$. 有趣的是, $\mathrm{H}_{2} \mathrm{~L}$ 具有很弱的反饱和吸收 $(\beta=1.8 \times$ $\left.10^{-12} \mathrm{~m} \cdot \mathrm{W}^{-1}\right)$, 但在 $\mathrm{H}_{2} \mathrm{~L}$ (1.0 equiv.)溶液中加入三乙胺 (2.0 equiv.), 其 $\beta$ 值增加到 $4.5 \times 10^{-12} \mathrm{~m} \cdot \mathrm{W}^{-1}$, 几乎是 $\mathrm{H}_{2} \mathrm{~L}$ 的三倍(图 S6). 这是因为三乙胺的加入中和了溶液 中的质子, 从而使偶氮单元的共轭程度得到了恢复. 偶 氮基团共轭程度的恢复不仅提高了其三阶 NLO 的性能, 还打开了其光开关的性能. 此外, 照射后的(TEA $)_{2} \mathrm{~L}$ 的 $\beta$ 值比 $\mathrm{H}_{2} \mathrm{~L}$ 的 $\beta$ 值提高了一个数量级.
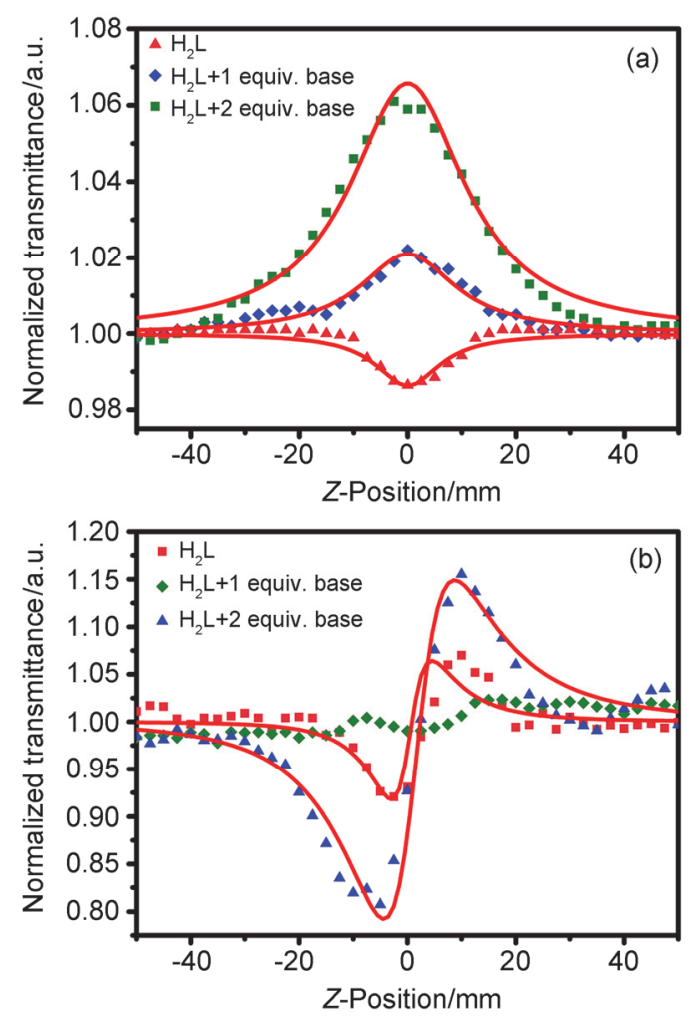

图 $5 \mathrm{H}_{2} \mathrm{~L}$ 在 $0 、 1.0$ 和 2.0 equiv. 三乙胺作用下的 NLO 吸收特性(a)和 NLO 折射特性(b)

Figure 5 NLO absorption properties (a) and NLO refraction properties (b) of $\mathrm{H}_{2} \mathrm{~L}$ with $0,1.0$ and 2.0 equiv. of triethylamine upon $\mathrm{UV}$ irradiation

虽然 $\mathrm{H}_{2} \mathrm{~L}$ 由于其自身的结构特点没有表现出光控
的转换行为, 但经过除去质子(有机基团及碱)可以有效 地调控其光控 NLO 开关特性. 重要的是，调节后的材料 在紫外光照射下展示出完全相反的三阶 NLO 性质. 移 去 UV 后，在黑暗条件下，顺式 $\left(\mathrm{CH}_{3}\right)_{2} \mathrm{~L}$ 和顺式(TEA $)_{2} \mathrm{~L}$ 可以逐渐转变成反式构型, 同时还伴随着反饱和吸收和 自散焦折射行为的出现. 为了研究这两种材料是否可以 作为可逆光控开关, 我们将这两种材料在反式和顺式构 型之间重复切换 6 次(图6), 实验结果表明了它们具有良 好的抗疲劳性能, 这也为光控三阶 NLO 开关的应用奠 定了基础.
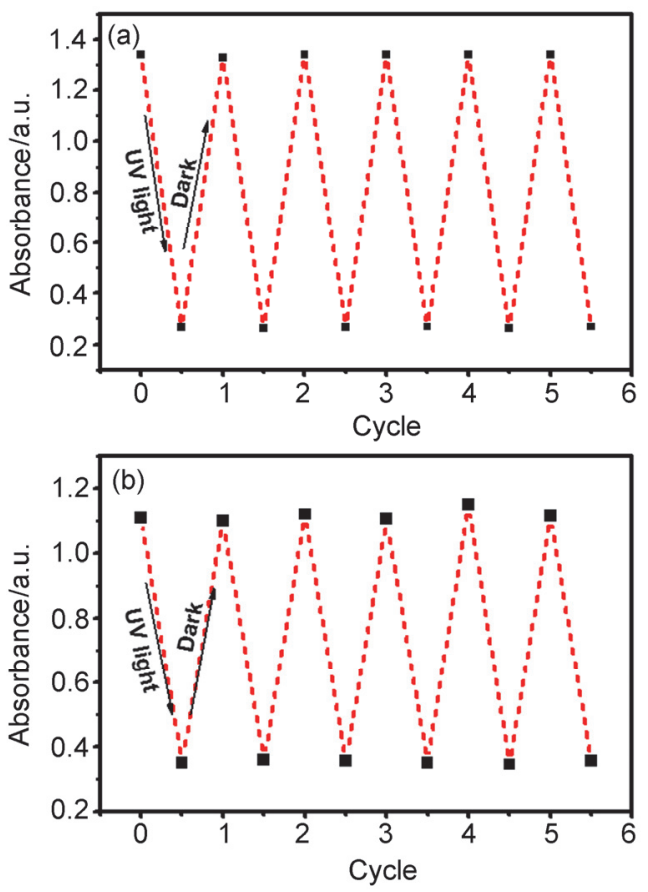

图 $6\left(\mathrm{CH}_{3}\right)_{2} \mathrm{~L}(\mathrm{a})$ 和 $(\mathrm{TEA})_{2} \mathrm{~L}$ (b) 在紫外光照射下和在黑暗中交替作用 的抗痛劳性实验

Figure 6 Fatigue resistance of $\left(\mathrm{CH}_{3}\right)_{2} \mathrm{~L}$ (a) and (TEA) $)_{2} \mathrm{~L}$ (b) upon UV light irradiation and standing in the dark alternatively

\section{3 结论}

基于具有光响应偶氮苯基团报道了一种独特的材 料 $\mathrm{H}_{2} \mathrm{~L}$. 该材料通过引入有机基团和碱，可以有效地将 其调控成为优异的光控三阶 NLO 开关. 调节后的材料 在光照前后展示出一个优异的三阶 NLO 性质的转变. 光照之前, 材料处于反式构型并展示出反饱和吸收和自 散焦特性; 光照之后, 材料转化为顺式构型并展现出饱 和吸收和自聚焦行为. 这项工作为后续设计光控三阶 NLO 开关材料提供了更多的选择.

\section{4 实验部分}

\section{1 试剂和仪器}

所有的化学品和溶剂都是直接购买和使用, 没有进 一步的净化. 4-氨基偶氮苯盐酸盐和 5-甲基间苯二甲酸 
购自萨恩化学技术(上海)有限公司. 其他溶剂购自国药 集团化学试剂北京有限公司. ${ }^{1} \mathrm{H}$ NMR 谱图在 $600 \mathrm{MHz}$ Bruker Avance-600 光谱仪测定. 溶液的紫外-可见吸收 光谱在室温条件下使用 JASCOV-750 分光光度计测量. C, H 和 N 元素分析在 FLASH EA 1112 元素分析仪进行. 傅立叶变换红外光谱用 Bruker Tensor 27 分光光度计进 行测定.

\section{2 非线性测试}

用标准皮秒顶帽 Z 扫描技术在开孔模式和闭孔模 式下测量了所有样品的三阶 NLO 吸收和折射特性. 采 用锁模 Q 开关 YAG 激光器(PW-1064-1H, 北京国科世纪 激光技术有限公司), 其提供了的激光波长为 $532 \mathrm{~nm}$,

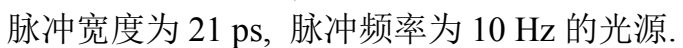

\section{3 晶体结构测定}

$\left(\mathrm{CH}_{3}\right)_{2} \mathrm{~L}$ 的数据在 $293 \mathrm{~K}$ 使用带有 $\mathrm{Mo} \mathrm{K \alpha}(\lambda=$ $0.071073 \mathrm{~nm}$ )的 Bruker D8 VENTURE 单晶衍射仪收集. 利用 SAINT 程序控制洛仑兹衍射数据的积分、偏振效 应和强度校正. 利用 SADABS 程序进行半经验吸收校 正. 利用 SHELXL-2014 晶体学软件包, 基于 $F^{2}$ 的全矩 阵最小二乘技术对结构进行求解和细化. 用骑乘模型几 何生成 $\mathrm{H}$ 原子, 并细化各向同性. $\left(\mathrm{CH}_{3}\right)_{2} \mathrm{~L}$ 的结晶学数据 见表 $\mathrm{S} 1$. 表 $\mathrm{S} 2$ 列出了 $\left(\mathrm{CH}_{3}\right)_{2} \mathrm{~L}$ 所选择的键长和键角. $\left(\mathrm{CH}_{3}\right)_{2} \mathrm{~L}(\mathrm{CCDC} 1963944)$ 的晶体学数据已被登记在剑桥 晶体学数据中心, 可通过网址 www.ccdc.cam.ac.uk/ data_request/cif 免费获得.

\section{4 化合物的合成}

4.4.1 (E)-5-(N-4-偶氮苯基)氨甲基间苯二甲酸 $\left(\mathrm{H}_{2} \mathrm{~L}\right)$ 的 合成

采用文献 ${ }^{[43]}$ 报道的方法合成了 5 -溴甲基间苯二甲 酸二甲酯. 将 4-氨基偶氮苯盐酸盐 $(0.4674 \mathrm{~g}, 2 \mathrm{mmol})$ 、 5 -溴甲基间苯二甲酸二甲酯 $(0.8580 \mathrm{~g}, 3 \mathrm{mmol})$ 和 $\mathrm{Na}_{2} \mathrm{CO}_{3}(0.3180 \mathrm{~g}, 3 \mathrm{mmol})$ 溶于 $20 \mathrm{~mL} N, N$-二甲基甲酰 胺 $(\mathrm{DMF})$ 中, 在 $120{ }^{\circ} \mathrm{C}$ 下搅拌 $6 \mathrm{~h}$. 待反应完全后冷却 到室温, 水/二氯甲烷洗反应溶液, 分离有机相, 无水 $\mathrm{Na}_{2} \mathrm{SO}_{4}$ 干燥, 旋干, 用柱色谱提纯(石油醚/乙酸乙酯, $V: V=20: 1)$, 所得产物为 5-(N-4-偶氮苯基)氨甲基间 苯二甲酸二甲酯. 将纯产物 $(0.4030 \mathrm{~g}, 1 \mathrm{mmol})$ 溶于四氢 呋喃(THF, $5 \mathrm{~mL})$ 与水 $(2 \mathrm{~mL})$ 的混合溶液中, 在搅拌后加 入 $3 \mathrm{~mL} \mathrm{NaOH}$ 水溶液 $\left(1 \mathrm{~mol} \cdot \mathrm{L}^{-1}\right)$. 反应液在 $65{ }^{\circ} \mathrm{C}$ 下摚 拌 $12 \mathrm{~h}$, 减压蒸馏有机溶剂. 将剩下的混合溶液用盐酸 调至 $\mathrm{pH}=1$ 时, 产生大量的红棕色沉淀, 将沉淀过滤后 放入真空干燥箱中得到固体 $\mathrm{H}_{2} \mathrm{~L}$ (Scheme $\mathrm{S} 1$ ). 产率 $62 \% .{ }^{1} \mathrm{H}$ NMR (DMSO- $\left.d_{6}, 600 \mathrm{MHz}\right) \delta: 8.37$ (s, 1H), 8.19 (s, 2H), $7.73(\mathrm{dd}, J=18.4,7.9 \mathrm{~Hz}, 4 \mathrm{H}), 7.51$ (t, $J=7.2 \mathrm{~Hz}$, 2H), 7.42 (t, $J=7.0 \mathrm{~Hz}, 1 \mathrm{H}), 6.75(\mathrm{~d}, J=8.2 \mathrm{~Hz}, 2 \mathrm{H}), 4.57$ $(\mathrm{s}, 2 \mathrm{H}) ;{ }^{13} \mathrm{C}$ NMR (151 MHz, DMSO) $\delta: 167.02,152.43$, $152.14,143.32,141.24,132.58,131.93,129.95,129.69$,
$129.19,126.16,122.03,113.05,45.92 ;$ IR $(\mathrm{KBr}) v$ : $3177.18,2999.73,1719.39,1599.57,1382.22,1175.56$, 822.97, 758.64, 683.30, 519.16 $\mathrm{cm}^{-1}$; HRMS calcd for $\mathrm{C}_{21} \mathrm{H}_{18} \mathrm{~N}_{3} \mathrm{O}_{4}(\mathrm{M}+\mathrm{H})^{+}$376.1296, found 376.1286. Anal. calcd for $\mathrm{C}_{21} \mathrm{H}_{17} \mathrm{~N}_{3} \mathrm{O}_{4}$ : C 67.19, H 4.56, $\mathrm{N} \mathrm{11.19;} \mathrm{found} \mathrm{C}$ $66.45, \mathrm{H} 4.41, \mathrm{~N} 10.89$.

\subsection{2 (E)-5-( $N$-4-偶氮苯基)氨甲基间苯二甲酸二甲酯} $\left[\left(\mathrm{CH}_{3}\right)_{2} \mathrm{~L}\right]$ 的合成

$\left(\mathrm{CH}_{3}\right)_{2} \mathrm{~L}$ 的合成方法与 $\mathrm{H}_{2} \mathrm{~L}$ 的合成方法相同. 以 4氨基偶氮苯盐酸盐为原料, 收率 $65 \%$. ${ }^{1} \mathrm{H}$ NMR $\left(\mathrm{DMSO}-d_{6}, 600 \mathrm{MHz}\right) \delta: 8.38(\mathrm{~s}, 1 \mathrm{H}), 8.24(\mathrm{~s}, 2 \mathrm{H}), 7.74$ (d, $J=7.9 \mathrm{~Hz}, 2 \mathrm{H}), 7.70$ (d, $J=8.6 \mathrm{~Hz}, 2 \mathrm{H}), 7.51$ (t, $J=7.6$ $\mathrm{Hz}, 2 \mathrm{H}), 7.42$ (t, $J=7.2 \mathrm{~Hz}, 2 \mathrm{H}), 6.74(\mathrm{~d}, J=8.7 \mathrm{~Hz}, 2 \mathrm{H})$, $4.58(\mathrm{~d}, J=6.0 \mathrm{~Hz}, 2 \mathrm{H}), 3.89$ (s, 6H); ${ }^{13} \mathrm{C} \mathrm{NMR}(151 \mathrm{MHz}$, DMSO) $\delta: 165.86,152.88,151.99,143.73,142.13,132.63$, $130.85,130.03,129.63,128.74,125.52,122.24,112.61$, 52.93, 45.70; IR (KBr) v: 3390.80, 1730.68, 1599.46, 1433.17, 1138.22, 825.60, 746.63, 688.26, $547.09 \mathrm{~cm}^{-1}$; HRMS calcd for $\mathrm{C}_{23} \mathrm{H}_{22} \mathrm{~N}_{3} \mathrm{O}_{4}(\mathrm{M}+\mathrm{H})^{+}$404.1605, found 404.1611. Anal. calcd for $\mathrm{C}_{23} \mathrm{H}_{21} \mathrm{~N}_{3} \mathrm{O}_{4}$ : C 68.47, $\mathrm{H} 5.25, \mathrm{~N}$ 10.42; found C 68.46, H 5.44, N 9.95.

\section{4 .3 滴定实验}

为了研究 $(E)-5-(N-4$-偶氮苯基)氨甲基间苯二甲酸 盐的三阶 $\mathrm{NLO}$ 开关的行为, 所以将 $\mathrm{K}_{2} \mathrm{CO}_{3}, \mathrm{Na}_{2} \mathrm{CO}_{3}$, $\mathrm{Cs}_{2} \mathrm{CO}_{3}, \mathrm{Cd}(\mathrm{Ac})_{2}, \mathrm{NH}_{3} \cdot \mathrm{H}_{2} \mathrm{O}$ 和三乙胺等分别添加到 $\mathrm{H}_{2} \mathrm{~L}$ 的 DMSO 溶液中以获取脱质子产物 $\mathrm{X}_{2} \mathrm{~L}$ (此时 $\mathrm{X}$ 是指一 价的阳离子).

\section{References}

[1] Delaire, A. J.; Nakatani, K. Chem. Rev. 2000, 100, 1817.

[2] Green, K. A.; Cifuentes, M. P.; Corkery, T. C.; Samoc, M.; Humphrey, M. G. Angew. Chem., Int. Ed. 2009, 48, 7867.

[3] Castet, F.; Rodriguez, V.; Pozzo, J. L.; Ducasse, L.; Plaquet, A.; Champagne, B. Acc. Chem. Res. 2013, 46, 2656.

[4] Xu, W. J.; Shao, Z. C.; Han. Y. B.; Wang, W.; Song, Y. L.; Hou, H. W. Dyes Pigm. 2018, 152, 171.

[5] Green, K. A.; Cifuentes, M. P.; Samoc, M.; Humphrey, M. G. Coord. Chem. Rev. 2011, 255, 2530.

[6] Champagne, B.; Plaquet, A.; Pozzo, J. L.; Rodriguez, V.; Castet, F. J. Am. Chem. Soc. 2012, 134, 8101.

[7] Feng, Q.; Li, Y. Y.; Shi, G.; Wang, L. L.; Zhang, W. J.; Li, K.; Hou, H. W.; Song, Y. L. J. Mater. Chem. C 2016, 4, 8552.

[8] Beaujean, P.; Bondu, F.; Plaquet, A.; Garcia-Amorós, J.; Cusido, J.; Raymo, F. M.; Castet, F.; Rodriguez, V.; Champagne, B. J. Am. Chem. Soc. 2016, 138, 5052.

[9] Boixel, J.; Guerchais, V.; Bozec, H. L.; Chantzis, A.; Jacquemin, D.; Colombo, A.; Dragonetti, C.; Marinotto, D.; Roberto, D. Chem. Commun. 2015, 51, 7805 .

[10] Dhammika Bandara, H. M.; Burdette, S. C. Chem. Soc. Rev. 2012, $41,1809$.

[11] Chu, Z.; Han, Y.; Bian, T.; De, S.; Král, P.; Klajn, R. J. Am. Chem. Soc. 2019, 141, 1949.

[12] Natali, M.; Giordani, S. Chem. Soc. Rev. 2012, 41, 4010.

[13] Zakrevskyy, Y.; Richter, M.; Zakrevska, S.; Lomadze, N.; von Klitzing, R. Adv. Funct. Mater. 2012, 22, 5000.

[14] Chen, L.; Tang, X.; Jia, K.; Tang, X. Z. Chin. J. Org. Chem. 2016 36, 2197. (陈垒, 唐翔, 贾坤, 唐先忠, 有机化学, 2016, 36, 2197.)

[15] Xu, W. J.; Wang, W.; Li, J. X.; Wu, Q.; Zhao, Y. J.; Hou, H. W.; 
Song, Y. L. Dyes Pigm. 2019, 160, 1

[16] Chen, L.; Bo, S. H.; He, Y. L.; Chen, Z.; Liu, X. H.; Zhen, Z. Chin. J. Org. Chem. 2017, 37, 2263. (陈璐, 薄淑晖, 和延岭, 陈卓, 刘 新厚, 甄珍, 有机化学, 2017, 37, 2263.)

[17] Qu, M.; Liu, M. M.; Liu, J.; Zhang, X. M. Chin. J. Chem. 2014, 32, 1259.

[18] Xie, Q.; Shao, Z. C.; Zhao, Y. J.; Yang, L. P.; Wu, Q.; Xu, W. J.; Li, K.; Song, Y. L.; Hou, H. W. Dyes Pigm. 2019, 170, 107599.

[19] Li, Q. Y.; Chi, Z.; Li, T. F.; Ran, X.; Guo, L. J. Opt. Express 2017, 25,11503

[20] Liaros, N.; Couris, S.; Maggini, L.; Leo, F. D.; Cattaruzza, F.; Aurisicchio, C.; Bonifazi, D. ChemPhysChem 2013, 14, 2961.

[21] Szymański, W.; Beierle, J. M.; Kistemaker, H. A. V.; Velema, W. A.; Feringa, B. L. Chem. Rev. 2013, 113, 6114.

[22] Xue, X. N.; Wang, H. R.; Han, Y. B.; Hou, H. W. Dalton Trans 2018 , $47,13$.

[23] Lovrien, R.; Pesheck, P.; Tisel, W. J. Am. Chem. Soc. 1974, 96, 244.

[24] Klotz, I. M.; Fiess, H. A.; Chen Ho, J. Y.; Mellody, M. J. Am. Chem. Soc. 1954, 76, 5136.

[25] Nihei, M.; Kurihara, M.; Mizutani, J.; Nishihara, H. J. Am. Chem. Soc. 2003, 125, 2964.

[26] Beharry, A. A.; Woolley, G. A. Chem. Soc. Rev. 2011, 40, 4422.

[27] Wu, Z.; Xue, R.; Xie, M.; Wang, X.; Liu, Z.; Drechsler, M.; Huang, J.; Yan, Y. J. Phys. Chem. Lett. 2018, 9, 163.

[28] Joshi, N. K.; Fuyuki, M.; Wada, A. J. Phys. Chem. B 2014, 118, 1891 .

[29] Wettermark, G.; Langmuir, M. E.; Anderson, D. G. J. Am. Chem. Soc. 1965, 87, 476.

[30] Beharry, A. A.; Sadovski, O.; Woolley, G. A. J. Am. Chem. Soc. 2011, 133, 19684.
[31] Zhou, H.; Xue, C.; Weis, P.; Suzuki, Y.; Huang, S.; Koynov, K.; Auernhammer, G. K.; Berger, R.; Butt, H. J.; Wu, S. Nat. Chem. 2017, 9, 145 .

[32] Zhang, W.; He, C. Y.; Xiao, X. W.; Song W. N.; Gao, Y. C.; Chen, Z M.; Dong, Y. L.; Wu, Y. Q.; Wang, Q. Chin. J. Chem. 2016, 34, 1006.

[33] Liang, P. X.; Mi, Y. S.; Duan, J. S.; Yang, Z.; Wang, D.; Cao, H.; He, W. L.; Yang, H. Chin. J. Chem. 2016, 34, 381.

[34] Niu, R. J.; Zhou, W. F.; Liu, Y.; Yang, J. Y.; Zhang, W. H.; Lang, J. P.; Young, D. J. Chem. Commun. 2019, 55, 4873.

[35] Zhang, C.; Song, Y. L.; Wang, X. Coord. Chem. Rev. 2007, 251, 111.

[36] Chen, Q. F.; Zhao, X.; Liu, Q.; Jia, J. D.; Qiu, X. T.; Song, Y. L.; Young, D. J.; Zhang, W. H.; Lang, J. P. Inorg. Chem. 2017, 56, 5669.

[37] Zhao, Y. J.; Li, H. H.; Shao, Z. C.; Xu, W. J.; Meng, X. R.; Song, Y. L.; Hou, H. W. Inorg. Chem. 2019, 58, 4792 .

[38] Li, J. L.; Ding, G. H.; Niu, Y. Y.; Wu, L. Y.; Duan, H. Y.; Feng, F. J.; He, W. J. Chin. J. Org. Chem. 2018, 38, 931. (李建玲, 丁国华, 牛 燕燕, 吴禄勇, 段红叶, 冯华杰, 何文英, 有机化学, 2018, 38, 931.)

[39] Wu, X. Z.; Xiao, J. C.; Sun, R.; Jin, T. X.; Yang, J. Y.; Shi, G.; Wang, Y. X.; Zhang, X. R.; Song, Y. L. Adv. Opt. Mater. 2017, 5, 1600712.

[40] Hou, H. W.; Wei, Y. L.; Song, Y. L.; Mi, L. W.; Tang, M. S.; Li, L. K.; Fan, Y. T. Angew. Chem., Int. Ed. 2005, 44, 6067.

[41] Liang, P. X.; Li, Z. Q.; Mi, Y. S.; Yang, Z.; Wang, D.; Cao, H.; He, W. L.; Yang, H. J. Electron. Mater. 2015, 44, 2883.

[42] Xiao, Z. G.; Shi, Y. F.; Sun, R.; Ge, J. F.; Li, Z. G.; Fang, Y.; Wu, X. Z.; Yang, J. Y.; Zhao, M. G.; Song, Y. L. J. Mater. Chem. C 2016, 4, 4647.

[43] Kanoo, P.; Matsuda, R.; Sato, H.; Li, L.; Jeon, H. J.; Kitagawa, S. Inorg. Chem. 2013, 52, 10735.

(Zhao, C.) 